Les lasers et leurs applications scientifiques et médicales

\title{
Dynamique des lasers
}

\author{
P. Glorieux
}

Laboratoire de Spectroscopie Hertzienne associé au CNRS, Université des Sciences et Technologies de Lille, 59655 Villeneuve d'Ascq cedex, France

L'objectif de ce cours est de montrer comment les concepts et méthodes de la dynamique nonlinéaire peuvent apporter un éclairage simple et performant en physique des lasers. La considération des ordres de grandeur, et notamment des échelles de temps et d'espace associées aux différents phénomènes concernés permet de dégager une hiérarchie de modèles et de cerner les conditions d'apparition des divers comportements dynamiques (relaxation amortie ou entretenue, oscillations chaotiques...). Ce cours s'inscrit dans la continuité du cours précédent dont la lecture préalable est recommandée. Celui-ci discute principalement le comportement stationnaire du laser, on mettra par contre ici l'accent sur ses propriétés temporelles et spatiotemporelles.

\section{LES LIMITES DE L'ÉTUDE ; GRANDEURS CARACTÉRISTIQUES DES LASERS}

Un des modèles les plus simples de laser, celui des équations du bilan sera d'abord discuté et permettra d'illustrer le point de vue adopté en dynamique des systèmes. On montrera ensuite comment les équations établies dans le cours précédent peuvent être généralisées de manière à prendre en compte la diffraction et les pertes. Le modèle plus complet qui en résulte servira alors de base à une discussion de la dynamique des différentes classes de lasers.

Comme cela a été exposé par C. Fabre, le laser est un générateur de rayonnement cohérent par émission stimulée qui met essentiellement en jeu un milieu actif placé dans une cavité optique. Souvent et par suite de multiples raisons, cette émission n'est pas continue mais présente un comportement périodique ou même erratique : impulsions du laser à modes bloqués ou automodulation du laser à absorbant saturable par exemple. Pour expliquer de tels comportements, il convient d'introduire un modèle dynamique du laser, c'est à dire susceptible de décrire son évolution temporelle. Une telle modélisation requiert bien sûr un recensement correct des différents phénomènes concernés et une discussion de l'échelle de temps et éventuellement d'espace associée à chacun d'eux. C'est l'objet de la présente section. Le lecteur moins intéressé par les discussions des limites de ce chapitre pourra se reporter directement à l'encadré de conclusion.

\section{1 Échelles de temps (ou de fréquence) de la cavité}

En ce qui concerne la cavité, on distingue :

- l'intervalle de fréquence entre modes longitudinaux $\Delta v_{\mathrm{L}}$ 


$$
D v_{L}=\frac{c}{n L}
$$

pour une cavité en anneau de longueur $L$ remplie d'un milieu d'indice de réfraction $n, c$ est la vitesse de la lumière dans le vide. Pour une cavité Perot-Fabry de même longueur

$$
\Delta v_{L}=\frac{c}{2 n L}
$$

- l'intervalle entre modes transverses $\Delta v_{T}$ dont la valeur a été discutée au chapitre précédent pour différents types de cavité, présente un rapport simple avec $\Delta v_{L}$

$$
\Delta v_{T}=\Delta v_{L} \operatorname{Arccos} \frac{L}{z_{R}}
$$

où $\mathrm{z}_{\mathrm{R}}$ est longueur de Rayleigh définie au chapitre précédent.

- la largeur de résonance de la cavité $\Delta v_{c}$ est égale à l'inverse de la durée de vie $T_{c}$ des photons dans la cavité. Elle est liée de façon simple au taux de décroissance $\kappa$ du champ électrique dans la cavité

$$
2 \pi \Delta v_{c}=\Delta \omega_{c}=\frac{1}{T_{c}} \equiv 2 \kappa
$$

Dans les premières sections de ce chapitre, on se place dans le cas où $\Delta v_{c}<\Delta v_{T}<\Delta v_{L}$. Cette restriction sera levée dans la dernière partie du cours où l'on discutera la dynamique spatio-temporelle des lasers.

\section{2 Échelles de temps du milieu actif}

En ce qui concerne les échelles de temps relatives au milieu actif, on distingue essentiellement la largeur homogène $\gamma_{a b}$, inverse de la durée de vie du dipôle microscopique et la largeur inhomogène $\Delta \omega_{\text {inh }}$ caractérisant la largeur de la distribution des fréquences de résonance atomique $\omega_{0}$. Le comportement dynamique des lasers est aussi régi par l'inverse de la durée de vie des populations des niveaux atomiques que par souci de simplicité, l'on choisit tous égaux $\gamma_{a}^{-1}=\gamma_{b}^{-1} \equiv \gamma$. Par suite de la nature des phénomènes de relaxation, on peut d'ailleurs démontrer que $\gamma_{a b}>\gamma$. Toujours dans le souci d'explorer le modèle le plus simple possible, on se limite aussi dans ce chapitre à la modélisation des lasers à élargissement homogène, c'est à dire pour lesquels l'élargissement inhomogène $\Delta \omega_{i n h}$ est négligeable par rapport à $\gamma_{a b}$.

\subsection{Hiérarchie des échelles de temps}

La situation la plus simple correspond à celle du laser monomode en anneau pour lequel la cavité est plus sélective que le milieu actif, c'est à dire $\Delta v_{T}, \Delta v_{L} \gg \kappa$. Si la transmission des miroirs est suffisamment faible, la répartition du champ électromagnétique est alors uniforme dans la cavité. L'amplitude du champ électromagnétique est quasiment constante le long de l'axe de la cavité, c'est 1"'approximation du champ moyen". En pratique, la condition de laser monomode requiert aussi que les désaccords $\delta_{a}$ et $\delta_{c}$ entre la pulsation $\omega$ de ce champ et les pulsations de résonance atomique $\omega_{o}$ et de la cavité $\omega_{c}$ soient faibles devant les écarts entre modes de telle sorte qu'un seul mode soit excité à la fois. La hiérarchie des temps et pulsations est alors telle que

$$
\Delta \omega i_{n h}<<\gamma_{a}, \kappa, \gamma_{a b}, \delta_{a}, \delta_{c}<<\Delta v_{T}, \Delta v_{L}
$$


Pour de tels lasers monomodes, en anneau et à élargissement homogène, la dynamique est donc conditionnée par les valeurs relatives des trois paramètres $\gamma, \kappa$ et $\gamma_{a b}$, données du dispositif utilisé, les désaccords $\delta_{a}$ et $\delta_{c}$ pouvant en général être réglés expérimentalement.

On utilise ici le principe d'élimination adiabatique des variables rapides qui peut s'exprimer de façon lapidaire: "les variables lentes asservissent les variables rapides". Plus précisément on considère que ces dernières relaxent très rapidement vers leur état d'équilibre instantané et disparaissent donc de l'évolution du système à long terme qui est dominée par les seules variables lentes. La comparaison des ordres de grandeur relatifs des constantes de relaxation $\kappa$ du champ, $\gamma_{a b}$ de la polarisation et $\gamma$ des populations dans les lasers effectivement rencontrés conduit à distinguer trois classes de lasers :

- Lasers de classe A $\left(\kappa \ll \gamma, \gamma_{a b}\right)$. Les variables atomiques relaxent alors rapidement vers leur valeur d'équilibre et la dynamique du laser est régie par la seule équation du champ. $\mathrm{C}^{\prime}$ est le cas des lasers $\mathrm{He}-\mathrm{Ne}, \mathrm{Ar}^{+}$, colorant....

- Lasers de classe $\mathbf{B}\left(\gamma, \kappa \ll \gamma_{a b}\right)$. La relaxation de la polarisation est très rapide par rapport à celle des autres variables dynamiques (champ, populations) qui régissent donc seules la dynamique du laser. C'est le cas des lasers les plus couramment utilisés (diode, $\mathrm{CO}_{2}, \mathrm{YAG}$ ).

- Lasers de classe $\mathrm{C}\left(\gamma \approx \kappa \approx \gamma_{a b}\right)$. Quand toutes les variables évoluent à la même échelle de temps, on ne peut éliminer aucune d'elles, le modèle a la complexité maximale. C'est le cas des lasers infrarouge lointain à ammoniac par exemple.

En toute rigueur, les arguments développés ci-dessus ne sont valables que pour les lasers monomodes en anneau. Pour les autres situations (multimode, Perot-Fabry et/ou élargissement inhomogène), il convient d'effectuer prudemment l'élimination adiabatique des variables rapides 1,2 . En dépit de ces restrictions, la distinction des trois classes de lasers garde sa pertinence dans les cas plus complexes.

Comme on vient de le voir, les lasers les plus répandus sont de classe B. Leur comportement en régime monomode est donc décrit par le système d'équations couplées reliant la différence de population des deux niveaux et le champ ou la densité d'énergie électromagnétique introduits dans le cours de Claude Fabre (cours de C. Fabre précédent) et que nous analysons dans un point de vue de dynamique au paragraphe suivant.

\section{LE MODÈLE DES ÉQUATIONS DU BILAN}

\subsection{Rappel et passage en unités réduites}

Les équations du bilan relient la différence de population $N_{b a}$ des niveaux a et b et la densité d'énergie électromagnétique $u$ dans le milieu actif comme cela a été démontré au chapitre précédent

$$
\frac{d N_{b a}}{d t}=-2 B N_{b a} u-\gamma\left(N_{b a}-N_{b a}^{0}\right)
$$

1 Il peut être abusif d'éliminer adiabatiquement une variable sur la base des seuls ordres de grandeurs présentés ici. Il convient en fait de déterminer les modes propres dynamiques du système. Il se peut en effet que comme dans des pendules couplés, une combinaison de variables rapides donne naissance à une variable lente. La détermination des "modes propres" du système dynamique permet d'identifier variétés lentes et rapides et de procéder, en toute sécurité, à l'élimination adiabatique des variétés rapides.

2 La prise en compte des ondes stationnaires dans un laser à cavité Perot-Fabry, de l'élargissement inhomogène ou du caractère multimode de l'émission introduit de nouvelles échelles (spatio)-temporelles dans le modèle et nécessite donc elle aussi une approche spécifique de l'élimination adiabatique. 


$$
\frac{d u}{d t}=-\frac{u}{T_{c}}+\frac{B}{h \omega} N_{b a} u
$$

où $N_{b a}^{\circ}$ est l'inversion de population en champ nul, c'est à dire, en adoptant les notations du chapitre précédent,

$$
N_{b a}^{0}=N_{b}^{0}-N_{a}^{0}=N\left\{\left(\frac{\Lambda_{b}}{\gamma_{b}}\right)-\left(\frac{\Lambda_{a}}{\gamma_{a}}\right)\right\}
$$

(cf. éqs. $15-17$ cours de C. Fabre précédent) et $B=B_{21}=B_{12}$ est le coefficient d'Einstein. Dans l'équation de population (2.a), le premier terme décrit l'émission stimulée et le second les processus de pompage et de relaxation. Dans l'équation de la lumière (2.b), le premier terme traduit les pertes du laser (eq. 43 cours de C. Fabre précédent), transposées dans une échelle d'énergie et le second décrit la production de photons par émission stimulée, la production d'un photon s'accompagnant d'une réduction de deux unitéx de la différence de population.

Un changement de variables évident permet de passer aux variables adimensionnelles $m$ et $N$ décrivant respectivement l'intensité lumineuse et la différence de population

$$
\begin{aligned}
& \frac{d N}{d \tau}=A-N(m+1) \\
& \frac{d m}{d \tau}=G m(N-1)
\end{aligned}
$$

où $\tau$ est le temps en unités de $\gamma^{-1} . G=2 \kappa / \gamma=T_{1} / T_{C}$ fixe le rapport des échelles de relaxation des deux variables dynamiques $m$ et $N$.

En dépit de sa simplicité, le système (3) n'admet pas de solution analytique. Il permet cependant d'accéder à certaines propriétés physiques du laser. Ainsi on le résout facilement en régime stationnaire et on établit donc les solutions d'équilibre. On effectue ensuite une analyse de stabilité linéaire de ces solutions pour délimiter leur(s) domaine(s) d'(in)stabilité et déterminer le comportement dynamique du laser pour de petites variations autour de l'équilibre.

\subsection{Solutions stationnaires}

Les situations d'équilibre du laser correspondent aux solutions stationnaires des équations du laser, c'est à dire telles que les dérivées des variables soient nulles.

Le système (3) admet deux solutions stationnaires

$$
\begin{array}{lcc}
\text { - la solution triviale (ou OFF) } & m=0 & N=A \\
\text { - une solution non triviale (ou } O N) & m=A-1 & N=1
\end{array}
$$

La première correspond à l'absence d'émission $(m=0)$. L'inversion de population est alors évidemment égale à sa valeur en champ nul $A$..

La solution non triviale (Laser ON) n'a de sens physique que pour $A$ supérieur à 1 . L'émission laser n'existe que si le pompage dépasse un certain seuil pris comme référence. L'inversion de population sature alors à sa valeur au seuil $(N=1$, dans le système d'unités réduites choisi ici).

En résumé, sous le seuil $(A<1)$, seule la solution OFF existe, tandis qu'au delà de celui-ci $(A>1)$, deux solutions d'équilibre coexistent. Pour déterminer celle qui sera effectivement observée, on effectue une analyse de stabilité linéaire, ce qui est l'objet de la section suivante. 


\subsection{Analyse de stabilité linéaire}

L'analyse de stabilité linéaire consiste à déterminer l'évolution de petites variations autour des équilibres. Si l'équilibre est stable, ces variations s'amortissent avec le temps, elles divergent dans le cas d'un équilibre instable.

En pratique, on linéarise le système différentiel concerné au voisinage des solutions stationnaires (), c'est à dire que l'on pose

$$
\begin{aligned}
& m=\bar{m}+\varepsilon_{m} \\
& N=\bar{N}+\varepsilon_{N}
\end{aligned}
$$

où $\bar{m}$ et $\bar{N}$ sont les solutions stationnaires données en 2.2 et l'on ne garde dans les équations d'évolution que les termes du premier ordre en $\varepsilon$. Le système (3) prend alors la forme

$$
\begin{gathered}
\frac{d \varepsilon_{m}}{d t}=G \varepsilon_{m}(\bar{N}-1)+G \bar{m} \varepsilon_{N} \\
\frac{d \varepsilon_{N}}{d t}=-\bar{N} \varepsilon_{m} \quad-(\bar{m}+1) \varepsilon_{N}
\end{gathered}
$$

Ce système différentiel linéaire du premier ordre à coefficients constants se résout simplement. Remarquons toutefois qu'il n'est pas nécessaire de le résoudre explicitement car il suffit de connaître le signe des racines de son équation caractéristique pour établir la stabilité des solutions stationnaires. A titre d'exemple pour illustrer la méthode, on traite ici l'analyse de stabilité linéaire de la manière la plus générale. Pour cela, on pose

$$
\frac{d}{d t}\left[\begin{array}{l}
\varepsilon_{m} \\
\varepsilon_{N}
\end{array}\right]=(L)\left[\begin{array}{l}
\varepsilon_{m} \\
\varepsilon_{N}
\end{array}\right]
$$

$\varepsilon_{m}$ et $\varepsilon_{N}$ évoluent au voisinage de leurs valeurs d'équilibre sous forme d'une combinaison d'exponentielles dont les taux sont les racines $\lambda$ de l'équation caractéristique de la matrice $L$

$$
\operatorname{det}|L-\lambda I|=0
$$

Pour la solution triviale ( $\bar{m}=0, \bar{N}=A$ ), les valeurs propres sont respectivement - 1 et $G(A$ I). La seconde n'est négative que sous le seuil $(A<1)$ tandis que la première l'est toujours. La solution triviale n'est donc stable que sous le seuil et instable au delà.

L'équation caractéristique associée aux valeurs stationnaires de la solution non nulle

$$
\lambda^{2}+A \lambda+G(A-1)=0
$$

se résout facilement. En fait on peut établir directement le signe des deux racines: celles-ci sont négatives si leur somme $S=-A$ est négative et leur produit $P=G(A-1)$ positif, ce qui est le cas au delà du seuil $(A>1)$.

En résumé, la solution triviale (OFF) est stable au dessous du seuil, instable au delà et la solution non nulle $(\mathrm{ON})$ est stable dans tout son domaine d'existence, c'est à dire pour $A>1$.

L'ensemble des résultats de l'analyse de stabilité linéaire est schématisé à la Figure 1. 

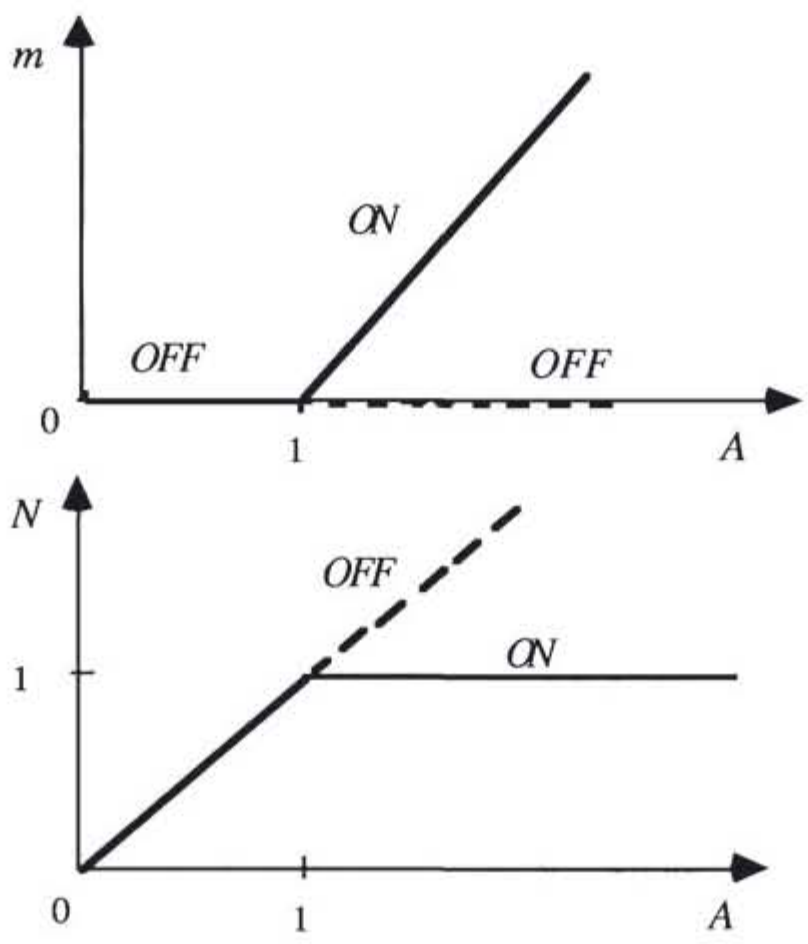

Figure 1 : Evolution de l'intensité m et de l'inversion de population réduites pour les deux solutions des équations du bilan. Traits pleins : solutions stables, pointillés : solutions instables.

L'écriture explicite des solutions de l'équation de stabilité du laser ON sous la forme

$$
\lambda_{ \pm}=\frac{-A \pm\left[A^{2}-4 G(A-1)\right]^{1 / 2}}{2}
$$

montre que comme dans la plupart des lasers $G$ est très supérieur à l'unité, la quantité sous le radical est négative et le retour à l'équilibre se fait de manière oscillatoire amortie avec une pulsation $\Omega_{r}=[G(A-1)]^{1 / 2}$.

Remarque: L'existence d'oscillations de relaxation amorties dans ces lasers laisse présager d'effets de résonance quand on modulera l'un des paramètres du laser. Il faut noter que cette résonance est d'origine purement dynamique, c'est à dire que la valeur de $\Omega_{r}$ n'est pas liée à une seule des variables mais qu'elle dépend à la fois des constantes de relaxation du champ $T_{c}$ et des populations $\gamma$. En unités physiques, $\Omega_{r}$ vaut en effet $\left[\gamma T_{c}(A-1)\right]^{I / 2}$. Ceci correspond à des oscillations dans le domaine $10^{4}-10^{5} \mathrm{~Hz}$ pour la plupart des lasers $\mathrm{CO}_{2}$ et YAG et $10^{9} \mathrm{~Hz}$ 
pour les diodes laser. Ces aspects de résonance apparaissent aussi dans l'analogie mécanique présentée en Annexe 4.

\subsection{Le seuil du laser considéré comme une bifurcation}

Il est intéressant de relever ici quelques propriétés du laser au voisinage du seuil. Celui-ci est marqué par l'échange de stabilité entre les deux solutions des équations du laser. C'est un exemple simple de bifurcation, c'est à dire d'une zone de paramètres associée à un changement qualitatif du comportement des solutions du problème considéré. Celui-ci est directement lié au changement de signe des coefficients de l'analyse de stabilité linéaire. Remarquons d'abord que ceux-ci étant continus, ils sont donc nuls au seuil. Or ces coefficients renseignent sur la rapidité de retour à l'équilibre du système. Les temps associés sont inversement proportionnels aux $\lambda$ et la dynamique du laser se ralentit dramatiquement au voisinage du seuil. Le laser au seuil présente en fait une "bifurcation transcritique" et comme tout système au voisinage d'une bifurcation présente l'effet de "ralentissement critique". On peut aussi montrer que la criticalité s'accompagne d'une augmentation dramatique des fluctuations.

Dans le modèle discuté ici, l'émission spontanée est négligée. Si celle-ci est incluse dans les équations, la bifurcation devient imparfaite et perd son caractère critique. Ce phénomène tout à fait négligeable dans les lasers $\mathrm{CO}_{2}$ peut devenir important dans des lasers comme les lasers à diode ou les lasers à fibre dopée en raison d'une collecte efficace de l'émission spontanée dans les structures guidantes. L'émission spontanée peut alors réduire de façon conséquente le ralentissement critique.

Nous verrons à la section 3.5 un autre type de bifurcation, la "bifurcation de Hopf". C"est l'objet de la théorie des bifurcations de recenser les différents types de bifurcation et d'en étudier les propriétés. Grâce aux apports de cette théorie, il est possible d'étudier analytiquement le comportement dynamique du laser au voisinage et même parfois loin des situations d'équilibre.

\subsection{Espace des phases}

Le comportement dynamique d'un système peut être représenté géométriquement dans un espace rapporté aux différentes variables, par exemple $\mathrm{E}, \mathrm{P}$ et $\mathrm{N}$ et que l'on nomme "espace des phases". L'état instantané du système y est représenté par un point dont les coordonnées sont les différentes valeurs prises à un instant donné par les variables relevantes. L'évolution du système considéré y est représentée par la succession de ces points qui forment la "trajectoire" dans l'espace des phases. Les états d'équilibre sont les points fixes de ces trajectoires.

Dans le cas des lasers de classe B modélisés par les équations du bilan, le système admet deux variables dynamiques $m$ et $N$ et l'espace des phases a deux dimensions. On peut alors représenter l'évolution du laser par une courbe dans le plan $(m, N)$. La figure 2 donne l'exemple d'une trajectoire associée à la mise en route du laser. L'état initial correspond à une inversion de population $\mathrm{N}_{\mathrm{O}}$ et une intensité quasi nulle $(N \cong 0)$ et le laser évolue de façon oscillatoire amortie vers l'état stationnaire final ( $m=A-1 ; N=1)$.

La dynamique des systèmes apporte un certain nombre de résultats qui limitent le type de comportement que l'on peut observer dans un laser suivant sa classe (voir annexe 1). En particulier pour les systèmes à une variable comme les lasers de classe $\mathrm{A}$, on ne peut obtenir que des points fixes, c'est à dire des intensités constantes. Pour les systèmes à deux variables, ici les lasers de classe $\mathrm{B}$, les comportements asymptotiques, c'est à dire en temps infini sont, en sus des points fixes, des évolutions périodiques associés à des cycles dans l'espace des phases $^{3}$. Enfin pour les lasers de classe $\mathrm{C}$, la trajectoire se déploie dans un espace à trois

3 Ceci résulte du théorème de Poincaré-Bendixon qui établit que les seuls comportements asymptotiques des systèmes à deux dimensions sont les points fixes et les cycles limites, c'est à dire des comportements purement périodiques. 
dimensions et l'on peut observer des comportements quasi périodiques, c'est à dire faisant intervenir deux fréquences incommensurables ou même au chaos déterministe.

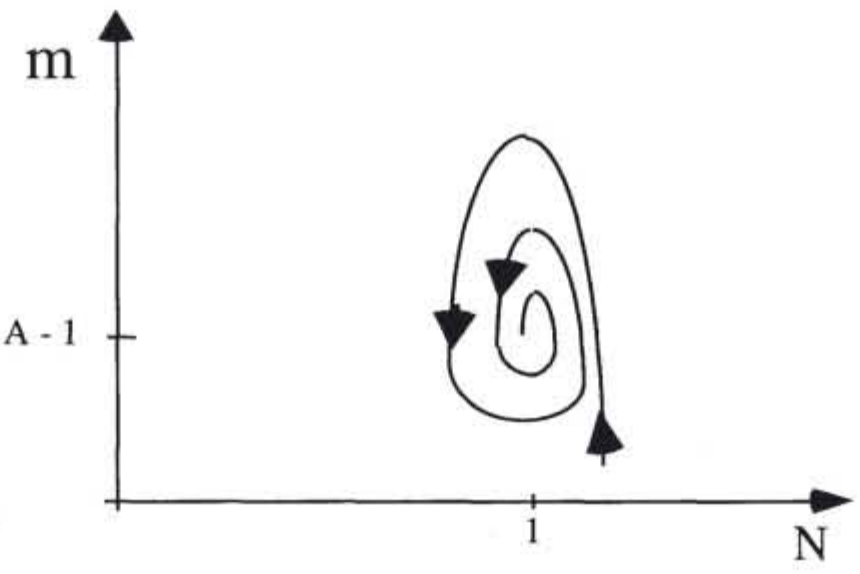

Figure 2 : Trajectoire dans l'espace des phases $(\mathrm{N}, \mathrm{m})$ d'un laser de classe B. Le laser évolue de manière oscillatoire amortie vers son équilibre

\section{6 Élimination adiabatique des populations. Démarrage de l'oscillation laser}

Dans les lasers pour lesquels la relaxation de la population est très rapide par rapport à l'amortissement du champ $(G<<I)$, les populations atteignent leur valeur d'équilibre instantané en un temps négligeable par rapport au champ. On peut alors remplacer la variable dynamique correspondante $N$ par sa valeur d'équilibre. C'est l'application aux lasers du "slaving principle" qui peut s'énoncer de façon lapidaire : "les variables lentes asservissent les variables rapides" ou de façon plus physique, les variables rapides atteignent en un temps très court leur position d'équilibre instantanée qui est régie par les variables lentes. Pratiquement dans un système comportant des variables évoluant à des échelles de temps différentes, on remplace dans les équations les variables rapides $\mathrm{X}_{\mathrm{R}}$ par leurs valeurs d'équilibre instantané, c'est à dire les solutions de

$$
\frac{d X_{R}}{d t}=0
$$

Dans le cas du laser dit de classe A, $\kappa<<\gamma$ et on peut donc remplacer la variable rapide $N$ par sa valeur d'équilibre et ne retenir que l'équation qui en résulte pour la variable lente $m$. On pose donc

$$
\frac{d N}{d t} \approx 0 \quad \text { soit } N=\frac{A}{m+1}
$$

Il faut bien noter qu'il s'agit là d'une valeur instantanée, $m$ restant une fonction lentement variable du temps. En réinjectant cette valeur de $N$ dans l'équation d'évolution de $m$, on en déduit l'équation dynamique de l'intensité

$$
\frac{d m}{d \tau}=\operatorname{Gm}\left[\frac{A}{m+1}-1\right]
$$


Si l'intensité est suffisamment faible on peut développer le dénominateur en puissances de $m$

$$
\frac{d m}{d \tau} \approx G m[A-1-A m]
$$

équation différentielle que l'on peut réćcrire, compte tenu de la proportionnalité de $m$ et de l'intensité $I$, sous la forme

$$
\frac{d I}{d t}=(\alpha-\beta I) I
$$

où $\alpha$ est le gain net par unité de temps et $\beta$ un coefficient qui traduit la saturation, c'est à dire la réduction du gain liée au champ laser.

L'intégration de (7) donne

$$
I(t)=\frac{\alpha I_{o} \exp \alpha t}{\alpha-\beta I_{o}+\beta I_{o} \exp \alpha t}=\frac{\alpha / \beta}{1+\left(\frac{\alpha}{\beta I_{o}}-1\right) \exp -\alpha t}
$$

Cette loi décrit la croissance de l'oscillation laser à partir d'une valeur initiale $I_{o}$. Aux temps courts, la croissance est exponentielle (en $\alpha^{-1}$ ) et elle sature aux temps longs comme le montre la Figure 3.

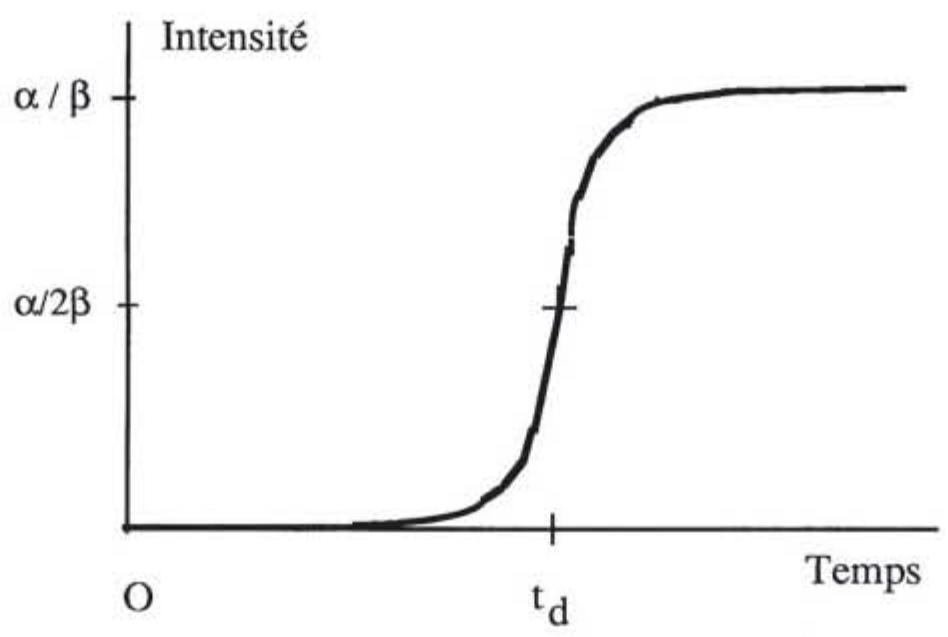

Figure 3 : Montée en oscillation d'un laser de classe A dans le cas où l'intensité initiale est très faible.

On calcule facilement le temps de délai $t_{d}$, temps nécessaire au laser pour atteindre la moitié de l'intensité stationnaire:

$$
t_{d}=\frac{I}{\alpha} \log \left(\frac{\alpha}{\beta I_{o}}-I\right) \approx \frac{I}{\alpha} \log \left(\frac{\alpha}{\beta I_{o}}\right)
$$


dans le cas du démarrage du laser sur son émission spontanée $\left(I_{o} \ll \frac{\alpha}{\beta}\right)$ et le temps de montée $(10 \%-90 \%), \mathrm{t}_{\mathrm{m}}$ prend une forme simple

$$
\mathrm{t}_{m}=\frac{2}{\alpha} \log 9
$$

De la mesure de ces deux quantités ( $t_{d}$ et $t_{m}$ ), on déduit immédiatement le gain $\alpha$ du laser et l'intensité initiale $I_{o}$. Dans la plupart des lasers émettant dans le visible, celle-ci est due aux fluctuations du vide, le laser déclenche alors sur un bruit dont on peut retrouver la statistique en inversant les données issues des mesures sur les délais. On suppose alors que l'équation d'évolution est purement déterministe, ce qui est contradictoire. Pour être cohérent, il convient d'établir l'équation de Fokker-Planck du laser. En fait le caractère aléatoire du champ n'apparaît que dans les tout premiers instants suivant le démarrage, la croissance en exp $(\alpha t)$ assurant très vite une densité de photons pour laquelle un modèle déterministe est valable.

L'équation d'évolution de $I$ possède une généralité dépassant largement le cadre de l'optique. L'écriture de l'équation (7) sous la forme $\frac{d I}{d t}=\alpha I-\beta I^{2}$ montre qu'on peut la considérer comme le début du développement en série d'une fonction s'annulant en zéro, décrivant donc un équilibre $(I=0)$ choisi comme origine. Il s'agit en fait du modèle le plus simple décrivant les phénomènes de croissance-saturation. La solution (8) décrit ici le passage d'un état d'équilibre instable $(I=0)$ à l'état d'équilibre stable $(I=\alpha / \beta)$ pour un système dynamique à une seule variable.

\section{3. ÉQUATIONS DE BLOCH-MAXWELL DU LASER}

L'objectif de cette section est d'établir un système d'équations différentielles exempt de certaines approximations utilisées dans la section précédente et touchant aux caractéristiques temporelles (par exemple $\gamma_{\mathrm{ab}} \gg \gamma_{\mathrm{a}}, \gamma_{\mathrm{b}}$ ) ou spatiales (diffraction négligeable) du laser. On est donc amené à reconsidérer l'écriture des équations du laser sur des bases plus larges que précédemment, en y incluant en particulier la dynamique de la polarisation diélectrique dans les équations du milieu (équations de Bloch) et l'influence des effets transverses dans les équations du champ (équations de Maxwell).

\section{1 Équations du champ}

Les équations de Maxwell a ppliquées à un milieu non magnétique obéissant à la loi d'Ohm $\overrightarrow{\mathbf{j}}=\sigma \overrightarrow{\mathbf{E}}$ permettent d'écrire

$$
\left.\overrightarrow{\operatorname{rot}}(\overrightarrow{\operatorname{rot}} \overrightarrow{\boldsymbol{E}})=\overrightarrow{\operatorname{rot}}\left(-\frac{\partial \vec{B}}{\partial t}\right)=-\mu_{0} \frac{\partial}{\partial t} \overrightarrow{\operatorname{rot}} \overrightarrow{(\vec{j}}+\frac{\partial \vec{D}}{\partial t}\right)
$$

En développant à l'aide des formules classiques

$$
\overrightarrow{\operatorname{rot}}(\overrightarrow{\operatorname{rot}} \overrightarrow{\boldsymbol{E}})=\operatorname{grad}(\operatorname{div} \overrightarrow{\boldsymbol{E}}) \cdot \overrightarrow{\Delta \vec{E}}
$$

pour un champ polarisé linéairement, et un milieu isotrope on aboutit donc à

$$
\frac{1}{c^{2}} \frac{\partial^{2} E}{\partial t^{2}}-\Delta E+\sigma \mu_{o} \frac{\partial E}{\partial t}=-\frac{1}{\varepsilon_{0} c^{2}} \frac{\partial^{2} P}{\partial t^{2}}
$$


On projette les champ et polarisation sur un mode propre de la cavité vide (cf. Ch. 1), c'est à dire que l'on sépare dans leur évolution les parties lentement variables de l'oscillation rapide à la fréquence $\omega_{\mathrm{c}}$ de la cavité. Soit

$$
\begin{aligned}
& E=\frac{1}{2} \hat{E}(r, z, t) \exp i\left(\omega_{c} t-k z\right)+c . c . \\
& P=\frac{1}{2} \hat{P}(r, z, t) \exp i\left(\omega_{c} t-k z\right)+c . c .
\end{aligned}
$$

Les effets de tirage de fréquence (frequency pulling) apparaissent alors dans la dépendance temporelle de $\hat{E}(r, z, t)$. On développe ensuite les dérivées par rapport à l'espace et au temps. A l'aide de l'approximation de l'amplitude et de la phase lentement variables ${ }^{4}$,

$$
\frac{\partial \hat{E}}{\partial z^{2}} \ll k \frac{\partial \hat{E}}{\partial z} ; \frac{\partial \hat{E}}{\partial t^{2}} \ll \omega_{c} \frac{\partial \hat{E}}{\partial t} \quad ; \quad \frac{\partial \hat{P}}{\delta t^{2}} \ll \omega_{c} \frac{\partial \hat{P}}{\delta t}
$$

et pour un milieu dilué $\left(\hat{\mathrm{P}} \ll \varepsilon_{0} \hat{\mathrm{E}}\right)$, il vient

$$
-k^{2} \hat{E}-2 i k \frac{\partial \hat{E}}{\partial z}-\frac{2 i \omega_{c}}{c^{2}} \frac{\partial \hat{E}}{\partial t}+\frac{\omega^{2} c}{c^{2}} \hat{E}+\Delta_{\perp} \hat{E}=-\mu_{O} \omega^{2} c \hat{P}
$$

A l'évolution du champ liée à la propagation et au couplage avec le milieu actif, il convient d'ajouter des termes reflétant l'interaction du champ avec la cavité. Dans un premier temps, on considère que celle-ci agit à la fois sur les pertes et en privilégiant certaines fréquences d'oscillation, en d'autres termes qu'elle se comporte comme un résonateur avec pertes. L'équation complète d'évolution du champ devient

$$
\frac{1}{c} \frac{\partial \hat{E}}{\partial t}+\frac{\partial \hat{E}}{\partial z}+\frac{\kappa}{c} \hat{E}-\frac{i c}{2 \omega_{c}} \Delta_{\perp} \hat{E}=\frac{i \omega_{c}}{2 \varepsilon_{0} c} \hat{P}
$$

où le coefficient de pertes peut prendre en compte celles liées au couplage soit

$$
\frac{\kappa}{c}=\frac{\sigma}{\varepsilon_{0}}+\frac{c}{L} \operatorname{Ln}\left(\frac{1}{R_{1} R_{2}}\right)
$$

et $R_{1}$ et $R_{2}$ sont les coefficients de réflexion des miroirs formant la cavité laser.

\section{2 Équations de Bloch}

Partant des équations de Bloch du système établies au chapitre précédent (Eq.7 du Cours de C. Fabre précédent)

$$
\frac{d \sigma_{a b}}{d t}=i \omega_{b} \sigma_{a b}+\frac{i E d}{2 \hbar} e^{i \omega_{c} t}\left(\sigma_{b b}-\sigma_{a a}\right)-\gamma_{a b} \sigma_{a b}
$$

on exprime les cohérences dans le référentiel tournant à la fréquence $\omega_{\mathrm{c}}$ du développement du champ (9)

$$
\sigma_{a b}=\hat{\sigma}_{a b}(t) e^{i \omega_{c} t} \quad \text { donc } \quad \frac{d \sigma_{a b}}{d t}=\left[\frac{d \hat{\sigma}_{b b}(t)}{d t}+i \omega_{c} \hat{\sigma}_{a b}\right] e^{i \omega_{c} t}
$$

et en ne retenant que les termes séculaires dans l'équation d'évolution de $\hat{q}_{a b}$

${ }^{4}$ Dans cette approximation, on suppose que l'amplitude et la phase de l'onde varient peu à l'échelle de la longueur d'onde et pendant une période optique. 


$$
\frac{d \hat{q}_{b b}}{d t}=i\left(\omega_{o}-\omega_{c}\right){\hat{\omega_{a}}}_{b}+i \frac{\hat{E} d}{2 \hbar}\left(\sigma_{b b}-\sigma_{a a}\right)-\gamma_{a b} \sigma_{a b}
$$

Pour la grandeur macroscopique $\hat{P}$, on aboutit donc à

$$
\frac{d \hat{P}}{d t}=i\left(\omega_{o}-\omega_{c}\right) \hat{P}-\gamma_{a b} \hat{P}+\frac{i \hat{E} d}{2 h}\left(\sigma_{b b}-\sigma_{a a}\right) N
$$

De même pour l'équation de population

$$
\frac{d\left(\sigma_{a a}-\sigma_{b b}\right)}{d t}=\frac{i E d}{h}\left[e^{i \omega_{c} t} \sigma_{b a}-e^{-i \omega_{c} t} \sigma_{a b}\right]-\gamma_{a} \sigma_{a a}+\gamma_{b} \sigma_{b b}+\Lambda_{a}-\Lambda_{b}
$$

où le premier terme décrit l'interaction lumière-matière et les termes suivants les effets du pompage et de la relaxation. Dans un modèle où $\gamma_{a}=\gamma_{b}=\gamma$, on obtient pour la densité de différence de population $N_{a b}=N\left(\sigma_{a a}-\sigma_{b b}\right)$

$$
\frac{d N_{a b}}{d t}=\frac{2 i}{h}\left[E^{*} P-E P^{*}\right]-\gamma\left[N_{a b}-N_{d b}^{(o)}\right]
$$

\section{3 Équations de Bloch-Maxwell du laser. Modèle de Haken-Lorenz}

Plusieurs changements de variables ont été proposés pour mettre ce système sous une forme adimensionnée. On adopte ici les notations de A. C. Newell et J. V. Moloney 5 . Il existe d'autres changements de variables menant à des équations parfois mieux adaptées à la résolution de certains problèmes (voir Annexe 2).

$$
\begin{aligned}
& \frac{\partial e}{\partial t}-i a \nabla_{\perp}^{2} e=-\sigma e+\sigma p \\
& \frac{\partial p}{\partial t}+(1+i \Omega) p=(r-n) e \\
& \frac{\partial n}{\partial t}+b n=\frac{1}{2}\left(e p^{*}+p e^{*}\right)
\end{aligned}
$$

où le temps est un temps réduit en unités de $\gamma_{\mathrm{ab}}$ et

$$
\sigma=\frac{\kappa}{\gamma_{a b}} \quad \Omega=\frac{\omega_{0}-\omega_{c}}{\gamma_{a b}}, \quad b=\frac{\gamma}{\gamma_{a b}} \quad r=\frac{\omega_{c} d^{2} N_{0}}{2 \varepsilon_{0} \hbar \kappa \gamma_{a b}}
$$

Si on néglige la dépendance transverse $(a=0)$, le système se réduit à un ensemble d'équations différentielles ordinaires pour lequel on peut appliquer la même démarche que celle développée pour le modèle des équations du bilan. Il prend la forme 6

$$
\partial_{t} e=-\sigma e+\sigma p
$$

5 A. C. Newell et J. V. Moloney, Nonlinear Optics, Addison-Wesley (Redwood City), 1992. Attention $\sigma$ par exemple, est maintenant le taux de décroissance du champ dans la cavité en unités de $\gamma_{a b}$ et non la conductivité comme dans la première partie du texte.

6 dans la suite on utilisera la notation allégée $\partial_{t}=\partial / \partial t$ 


$$
\begin{aligned}
& \partial_{t} p=(r-n) e-(1+i \Omega) p \\
& \partial_{t} n=-b n+\frac{1}{2}\left(e^{*} p+p^{*} e\right)
\end{aligned}
$$

Dans le cas de la cavité accordée $(\Omega=0)$, ce système est équivalent au système introduit par Lorenz en Physique Atmosphérique. Ce modèle a servi de paradigme pour l'étude du chaos et a fait l'objet de travaux extensifs 7 . La formulation ci-dessus a été introduite par Haken qui a justement noté que, de manière surprenante, ce modèle est manifestement inadéquat pour modéliser la circulation atmosphérique mais qu'il s'avère remarquablement efficace pour décrire certains lasers à deux niveaux.

\subsection{Solutions stationnaires et connexion aux équations du bilan}

Le système (16) admet deux solutions stationnaires

- la solution triviale $\dot{e}=\dot{p}=\dot{n}=0$ qui devient instable à $\mathrm{r}=\mathrm{r}_{\mathrm{C}}=1+\Omega^{2} /(\sigma+1)^{2}$ ou ce qui est équivalent

$$
\frac{\omega_{c} d^{2} N_{0}}{2 \varepsilon_{0} \kappa \hbar \gamma_{a b}}=1+\left\{\frac{\left(\omega_{0}-\omega_{c}\right)}{(\sigma+1) \gamma_{a b}}\right\}^{2}
$$

et l'on retrouve l'inversion de population au seuil $\mathrm{N}_{\mathrm{c}}$ du chapitre 1 .

- la solution lasante

$$
\left[\begin{array}{c}
\dot{e} \\
\dot{p} \\
\dot{n}
\end{array}\right]=\left[\begin{array}{c}
\sqrt{b\left(r-r_{c}\right)} e^{-i(\sigma \Omega) /(\sigma+1) t} \\
\left(1-i \frac{\Omega}{\sigma+1}\right) \sqrt{b\left(r-r_{c}\right)} e^{-i(\sigma \Omega) /(\sigma+1) t} \\
r-r_{c}
\end{array}\right]
$$

Avant de discuter la stabilité de cette solution, il est utile de comparer le résultat obtenu à celui du "bilan":

- Le modèle de Bloch-Maxwell incluant une description plus complète de l'interaction lumière-matière, fournit la dépendance du champ émis avec les désaccords entre les fréquences de cavité et atomique. Il donne de plus des informations sur la phase (fréquence) du champ émis.

- Le modèle des équations du bilan se déduit simplement de ce modèle en éliminant adiabatiquement $p$, c'est à dire en le remplaçant par sa valeur d'équilibre

$$
p=\frac{(r-n) e}{1+i \Omega}
$$

dans les équations de $e$ et $n$, soit

$$
\begin{gathered}
\partial_{l} e=-\sigma e\left[1-\frac{(r-n)}{1+i \Omega}\right] \\
\partial_{l} n=-b n+\mid e / 2 \frac{(r-n)}{1+\Omega^{2}}
\end{gathered}
$$

La densité d'énergie électromagnétique est proportionnelle à $/ e / 2$

$$
\partial_{l}|e|^{2}=e \partial_{l} e^{*}+e^{*} \partial_{l} e=-2 \sigma|e|^{2}+\frac{(r-n) \sigma e^{2}}{1+\Omega^{2}}
$$

7 voir par exemple le livre de C. Sparrow, The Lorenz equations: Bifurcations, Chaos and Strange Attractors (Springer-Verlag, New-York)(1982). 
Ce système différentiel est donc équivalent au système (2) moyennant un changement de variables simple.

\subsection{Analyse de stabilité linéaire et Instabilité de Haken-Lorenz}

La stabilité des solutions stationnaires du système complet peut être différente de celle du système d'équations du bilan. Dans le cas de la résonance exacte $(\Omega=0), \dot{e}$ et $\dot{p}$ peuvent être choisis réels et l'analyse de stabilité linéaire de la solution non nulle ramenée comme pour le système des équations du bilan, à un système différentiel linéaire. En ne gardant que les termes d'ordre $\varepsilon$,

$$
\begin{gathered}
\partial_{t} \varepsilon_{e}=-\sigma \varepsilon_{e}+\sigma \varepsilon_{p} \\
\partial_{l} \varepsilon_{p}=(r-\bar{n}) \varepsilon_{e}-\varepsilon_{p}-\bar{e} \varepsilon_{n} \\
\partial_{t} \varepsilon_{n}=-b \varepsilon_{n}+\dot{e} \varepsilon_{p}+\dot{p} \varepsilon_{e}
\end{gathered}
$$

où $\varepsilon_{e}, \varepsilon_{p}, \varepsilon_{n}$, sont les écarts par rapport aux solutions stationnaires $\dot{e}, \dot{p}, \dot{n}$. Soit sous forme matricielle

$$
\frac{\partial}{\partial t}\left[\begin{array}{l}
\varepsilon_{e} \\
\varepsilon_{p} \\
\varepsilon_{n}
\end{array}\right]=\left[\begin{array}{lll}
-\sigma & +\sigma & 0 \\
r-\bar{n} & -1 & -\dot{e} \\
\dot{p} & \dot{e} & -b
\end{array}\right]\left[\begin{array}{l}
\varepsilon_{e} \\
\varepsilon_{p} \\
\varepsilon_{n}
\end{array}\right]
$$

La résolution de ce système nécessite celle de son équation caractéristique

$$
\lambda^{3}+\lambda^{2}(b+\sigma+1)+\lambda b(r+\sigma)+2 \sigma b(r-1)=0
$$

Comme on l'a déjà vu, l'analyse de stabilité linéaire ne requiert pas la solution explicite du système car il suffit de connaître le signe des parties réelles des racines $\lambda_{i}$ de cette équation pour établir la stabilité des solutions stationnaires. Ces solutions ne sont stables que si les parties réelles $R e ́\left(\lambda_{i}\right)$ sont toutes négatives. Le critère de Routh-Hurwitz (voir Annexe 3) appliqué à une équation du type

$$
\lambda^{3}+a_{1} \lambda^{2}+a_{2} \lambda+a_{3}=0
$$
si

établit que les racines de cette équation ont des parties réelles toutes négatives si et seulement

$$
\begin{gathered}
a_{I}>0 \\
a_{1} a_{2}-a_{3}>0 \\
a_{3}>0 .
\end{gathered}
$$

Ces conditions appliquées au polynôme (21) prennent la forme

$$
\begin{gathered}
(b+\sigma+1)>0 \\
(b+\sigma+1) b(r+\sigma)-2 o b(r-1)>0 \\
2 \sigma b(r-1)>0
\end{gathered}
$$

La première condition est toujours vérifiée, la troisième indique que le laser doit être au dessus du seuil d'oscillation et seule la seconde condition apporte un élément nouveau. Remise en forme, elle indique que le laser devient instable si 


$$
r>\frac{b+1+3 \sigma}{\sigma-(b+1)}
$$

or la condition de seuil impose $r>1$, ce qui implique que linégalité

$$
\sigma>(b+1)
$$

soit vérifiée pour que le laser puisse devenir instable au delà du seuil. Compte tenu de la définition de $\sigma$ et de $b$, ceci peut s'écrire encore

$$
\kappa>\gamma
$$

L'instabilité de Haken-Lorenz ne peut donc se développer que dans un laser où le taux de pertes de la cavité est supérieur à la constante de relaxation des populations. C'est le concept de "mauvaise cavité", une "bonne cavité" étant par convention celle où le champ relaxe moins vite que les variables atomiques.

On peut aussi discuter analytiquement la nature de cette instabilité. Ainsi la déstabilisation des oscillations amorties du laser vers son état d'équilibre non nul se traduit mathématiquement par le fait que la partie réelle des deux racines complexes conjuguées change de signe et donc s'annule à la bifurcation. Pour établir la fréquence correspondante, il suffit d'écrire le polynôme caractéristique sous la forme imposant deux racines imaginaires pures

$$
\left(\lambda-\lambda_{0}\right)\left(\lambda^{2}+\omega^{2} H\right)
$$

et d'identifier à la forme(22). L'identité de cette équation avec l'équation caractéristique permet de retrouver la position de bifurcation(23). On établit aussi que les oscillations se produisent à la pulsation $\omega_{\mathrm{H}}=[\mathrm{b}(\mathrm{r}+\sigma)]^{1 / 2}$. Une telle bifurcation où le point attracteur se transforme d'une situation de foyer stable en foyer instable est appelée "bifurcation de Hopf" et constitue un autre cas générique de bifurcation.

L'instabilité de Lorenz-Haken est susceptible de se manifester dans un laser dont la dynamique est régie par le système de Lorenz (16) mais n'apparaît pas dans le système des équations du bilan (3), ce qui exclut de fait tous les lasers qui comme les lasers monomodes $\mathrm{CO}_{2}$ et YAG sont correctement décrits par ces équations du bilan. A l'heure actuelle, seuls les lasers moléculaires infrarouge lointain comme le laser à ammoniac présentent l'instabilité de Haken-Lorenz.

On a vu à plusieurs reprises que l'élimination adiabatique de variables dynamiques comme la polarisation $\mathrm{P}$ ou l'inversion de population $\mathrm{N}$ permettait, dans le cas où les échelles de temps concernées s'y prêtaient, de simplifier les modèles requis. Au contraire la possibilité pour le laser d'osciller simultanément sur plusieurs modes transverses augmente singulièrement la complexité des modèles et engendre d'éventuelles instabilités supplémentaires. Alors que dans le cas monomode, des modèles ordinaires comme ceux donnés par les systèmes d'équations différentielles ordinaires (3) ou (16) s'avèrent souvent suffisants, la prise en compte des effets transverses conduit à des systèmes d'équations aux dérivées partielles du type (15).

\section{MODÉLISATION DES LASERS MULTIMODES}

Avant de modéliser de tels lasers, il convient de spécifier le nombre de modes intervenant dans la dynamique ou pour le moins d'en connaître un ordre de grandeur. Si seul un petit nombre de modes est concerné, on peut développer les variables intervenant dans les équations de BlochMaxwell sur la base fournie par les modes de la cavité vide et l'on aboutit à un ensemble d'équations différentielles ordinaires couplées dont le nombre augmente avec celui des modes mis en jeu. Cette approche devient rapidement impraticable même numériquement dès que plus de 100 modes entrent en compétition. La décomposition modale n'est alors plus pertinente et il convient de considérer dans ce cas les grandeurs physiques comme des variables continues de l'espace et du temps. Ces deux approches sont présentées succinctement dans cette section. On discute d'abord de la dynamique d'un laser de classe A bimode puis on établit les bases de la méthode des équations d'amplitude pour les lasers fortement multimodes au voisinage du seuil. 


\section{1 Un exemple de dynamique d'un laser faiblement multimode : le laser de classe $\mathrm{A}$ bimode}

Dans les lasers de classe A, populations et polarisation ont été éliminées adiabatiquement et la dynamique est régie par la seule équation d'évolution de l'intensité (cf.2.6), soit pour un laser monomode:

$$
\frac{d l}{d t}=(\alpha-\beta I) I
$$

Pour un laser bimode, on peut généraliser cette équation en tenant compte de la saturation croisée, c'est à dire de la réduction de gain sur un mode liée à la présence du champ dans l'autre mode. Le modèle le plus simple décrivant le laser bimode est alors de la forme

$$
\begin{aligned}
& \frac{d I_{1}}{d t}=\left(\alpha_{1}-\beta_{1} I_{1}-\theta_{12} I_{2}\right) I_{1} \\
& \frac{d I_{2}}{d t}=\left(\alpha_{2}-\beta_{2} I_{2}-\theta_{2 I} I_{1}\right) I_{2}
\end{aligned}
$$

où les termes $\theta_{12} I_{2}$ et $\theta_{2 I} I_{I}$ représentent la saturation du gain pour un mode liée à l'intensité existant dans l'autre (saturation croisée). Ces équations admettent trois types de solutions :

- la solution triviale

$$
I_{1}=I_{2}=0
$$

- les deux solutions monomodes $I_{1}=0 ; \quad I_{2}=\alpha_{2} / \beta_{2}$

- une solution bimode

$$
\begin{array}{ll}
\text { et } \quad I_{1}=\alpha_{1} / \beta_{1} ; I_{2}=0 \\
I_{1}=\frac{\alpha_{2} \theta_{12}-\beta_{2} \alpha_{1}}{\theta_{21} \theta_{12}-\beta_{1} \beta_{2}}=\frac{\alpha^{\prime}{ }_{1} / \beta_{1}}{1-C} \\
\text { avec } \quad \alpha_{1}^{\prime}=\alpha_{1}-\theta_{21} \alpha_{2} / \beta_{2} \text { et } C=\frac{\theta_{21} \theta_{12}}{\beta_{1} \beta_{2}}
\end{array}
$$

et pour $I_{2}$ l'expression correspondante par permutation des indices 1 et 2 .

L'analyse de stabilité linéaire menée de manière classique permet de déterminer la stabilité de ces solutions. Pour chaque couple de solutions, on pose

$$
I_{i}=I_{i}^{s}+\varepsilon_{i} \quad i=1 \text { ou } 2
$$

où les $I_{i}^{s}$ sont les solutions stationnaires définies ci-dessus. On reporte dans les équations et on procède comme en 2.3 en se limitant aux termes du premier ordre en $\varepsilon$.

En ce qui concerne la solution triviale, l'analyse est directe; elle montre que comme pour le laser monomode, la solution OFF est stable sous le seuil de chaque mode, c'est à dire pour $\alpha_{1}$, $\alpha_{2}<0$ et instable dans le cas où l'une de ces conditions n'est pas remplie.

La solution bimode est stable au delà du seuil de chaque laser à la condition que $\mathrm{C}$ soit supérieur à l'unité et instable dans le cas contraire.

Suivant la valeur des paramètres, le système au delà du seuil évolue vers la solution bimode ou vers l'une des solutions monomodes.

\section{2 Équation d'amplitude pour les lasers}

Pour des lasers fortement multimodes au voisinage du seuil du laser, il est possible de suivre la méthode des équations d'amplitude inspirée de l'hydrodynamique. Pour cela on procède en trois étapes :

- Étape 1 : on examine d'abord la stabilité de la solution non lasante et on détermine le seuil $\mathrm{r}_{\mathrm{C}}$ du paramètre de pompage à partir duquel d'autres solutions vont croître exponentiellement. 
On s'intéresse à la forme et à la structure des modes ${ }^{8}$ qui sont neutres ou faiblement instables ou stables au voisinage de $\mathrm{r}=\mathrm{r}_{\mathrm{c}}$. Cet ensemble de modes est appelé 1 '"ensemble actif" et tous les autres modes sont appelés "passifs" en ce sens que leurs amplitudes dépendront de l'amplitude $A$ des modes actifs.
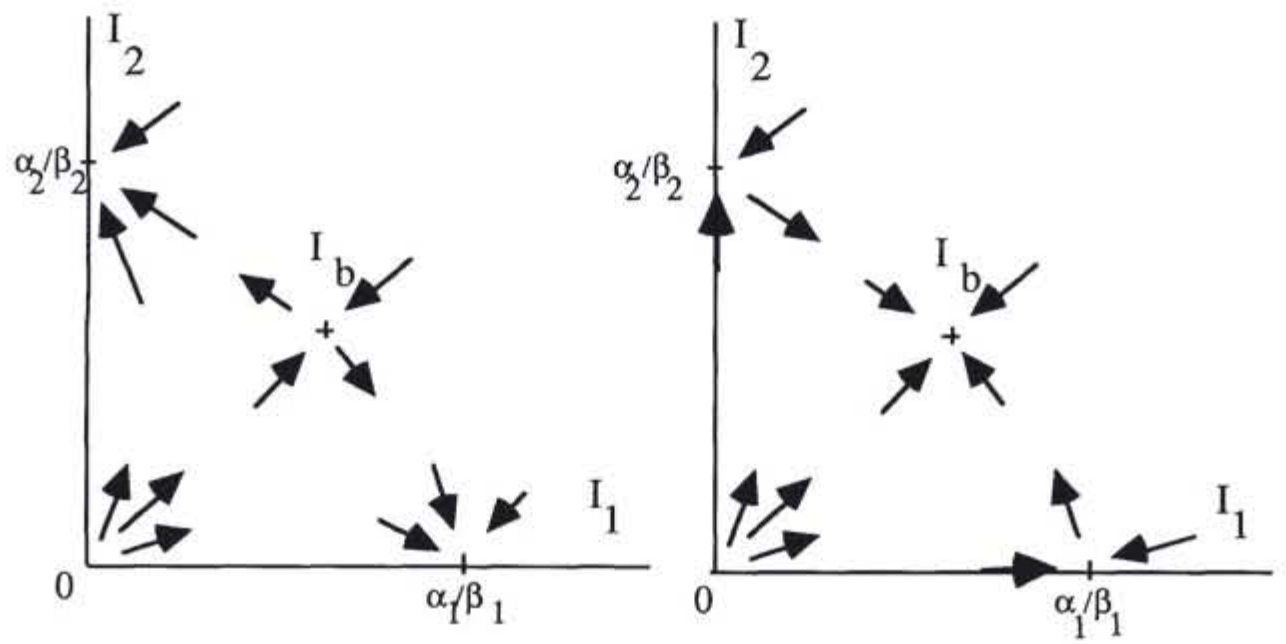

Figure 4 : Illustration des deux types de comportement du laser à deux modes (a) $C>1$, bistabilité entre deux solutions monomodes 1 et 2 (b) $\mathrm{C}<1$, seule la solution bimode est stable.

- Étape 2 : pour obtenir cette amplitude $A$, on développe le vecteur $\vec{v}=(A, P, n)$ en série par rapport au petit paramètre $\varepsilon=\mathrm{r}-\mathrm{r}_{\mathrm{C}}$

$$
\vec{v}=\varepsilon \vec{v}_{0}+\varepsilon \vec{v}_{1}+\varepsilon^{3} \vec{v}_{2}+\ldots
$$

où $\vec{v}$ est une combinaison linéaire de tous les modes actifs. Les corrections $\vec{v}_{1}, \vec{v}_{2}, \ldots$ satisfont à des équations inhomogènes et la condition de solvabilité de ces équations conduit à des équations pour les amplitudes des modes actifs.

- Étape 3 : l'analyse des équations pour ces modes actifs et leur impact sur les modes passifs mènent à la détermination de l'état du système.

Nous ne présentons ci-après que les deux premières étapes. L'analyse complète est disponible dans la littérature par exemple le livre de Moloney et Newell cité en ${ }^{5}$. En fait seule la première étape fournit déjà un certain nombre de propriétés des lasers et sera seule détaillée ici.

Étape 1 : Analyse de stabilité linéaire de la solution non lasante.

On linéarise les équations (15) autour de la solution $A=\mathrm{P}=\mathrm{n}=0$ en posant $A=0+A^{\prime}$, $\mathrm{P}=0+\mathrm{P}^{\prime}, \mathrm{n}=0+\mathrm{n}^{\prime}$ et on néglige les termes quadratiques dans les produits de $A^{\prime}, \mathrm{P}^{\prime}, \mathrm{n}^{\prime}$. On écrit

${ }^{8}$ Il s'agit ici des modes de type hydrodynamique (rouleaux,...) du champ laser dont l'expression est donnée plus loin et qui sont bien distincts des modes optiques de la cavité laser (modes d'Hermite-Gauss par exemple). 


$$
\left[\begin{array}{l}
A^{\prime} \\
P^{\prime} \\
n^{\prime}
\end{array}\right]=V_{0} e^{\lambda t+\vec{i} \vec{k} x} \quad \vec{k}=\left(k_{x}, k_{y}\right)
$$

et on montre que $V_{0}$ satisfait

$$
\Lambda V_{0}=\left[\begin{array}{lll}
\lambda+\sigma+i a k^{2} & -\sigma & 0 \\
-r & \lambda+1+i \Omega & 0 \\
0 & 0 & \lambda+b
\end{array}\right] V_{0}=0
$$

Le taux complexe de croissance des solutions est déterminé par det $\Lambda=0$ et l'on trouve

$$
\lambda=-b
$$

et

$$
\lambda^{2}+\left(\sigma+1+i a k^{2}+i \Omega\right) \lambda+\left(\sigma+i a k^{2}\right)(1+i \Omega)-\sigma r=0
$$

En posant $\lambda=\mu$-iv on peut exprimer le taux de croissance $\mu$ et la fréquence $v$ en fonction de $\mathrm{k}^{2}=\mathrm{kx}^{2}+\mathrm{ky}^{2}$ et du paramètre $\mathrm{r}$. Nous sommes surtout intéressés aux valeurs de $\mathrm{r}$ et $\mathrm{k}^{2}$ pour lesquelles le système se déstabilise, c'est à dire pour lesquelles $\mu$ s'annule en passant de valeurs négatives (stables) à positives (instables). Pour ces valeurs, $\mu=0$ et on obtient facilement

$$
\begin{gathered}
n=\frac{\sigma \Omega+a k^{2}}{1+\sigma} \\
r=1+\frac{1}{(1+\sigma)^{2}}\left(\Omega-a k^{2}\right)^{2}
\end{gathered}
$$

Le graphe de r en fonction de k (Figure 5) est appelé "courbe de stabilité neutre". Pour les couples $(r, k)$ situés au dessus de la courbe, la solution non lasante est instable. On voit que pour $\Omega<0$, le mode $\mathrm{k}=0$ est le plus instable tandis que quand $\Omega>0$, les modes les plus instables sont tels que $k= \pm \sqrt{\Omega / a}$.

Il est maintenant possible d'identifier les modes actifs au voisinage de $r=r_{c}$. Par suite de la dépendance transverse $(\mathrm{x}, \mathrm{y})$, il $\mathrm{y}$ a une infinité de tels modes. Pour $\mathrm{r}>\mathrm{r}_{\mathrm{c}}$, tous les modes $\mathrm{k}$ inscrits dans un cercle ou un anneau engendré en tournant les figures autour de l'axe r sont "actifs". Pour déterminer comment ces modes interagissent, on procède comme à une analyse nonlinéaire au voisinage de $\mathrm{r}=\mathrm{r}_{\mathrm{c}}$.

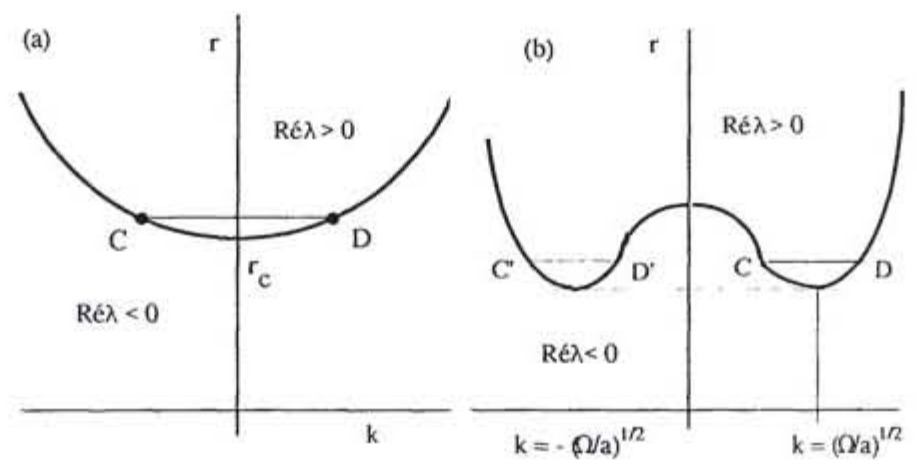

Figure 5 : Courbes de stabilité neutre. (a) $\Omega<0$. (b) $\Omega>0$. 


\section{Étape 2: Établissement de l'équation d'amplitude}

Théoriquement toutes les fonctions de $\mathrm{x}, \mathrm{y}$ et $\mathrm{t}$ bornées quand $\mathrm{x}^{2}+\mathrm{y}^{2}$ tend vers l'infini peuvent être représentées par des combinaisons linéaires des modes de Fourier $\mathrm{e}^{\mathrm{ikx}}, \mathbf{k}=\left(\mathrm{k}_{\mathrm{x}}\right.$, $\mathrm{k}_{\mathrm{y}}$ ). Cependant, quand

$$
r=r_{c}+R, \quad 0<R<<1
$$

on voit sur la figure que seuls les modes $\mathrm{e}^{i \mathbf{k x}}$ dont le nombre d'onde $\mathrm{k}$ tombe dans la bande CD se développent, et la bande a une largeur d'ordre $\sqrt{R}$. Le vecteur d'onde $k$ s'inscrit dans un cercle centré sur l'origine. Alors on utilise le concept de paquet d'ondes et on écrit

$$
\vec{v}_{0}=\sqrt{R} B_{1}(x, y, t) e^{-i v_{c} t} V_{o}(1)
$$

En établissant la condition de solvabilité à l'ordre 2, c'est à dire pour $\vec{v}_{2}$, on aboutit à une équation standard de la dynamique nonlinéaire, l'équation de Ginzburg-Landau complexe

$$
B_{t}+c B_{z}-\gamma \nabla_{l}^{2} B=\alpha B-\beta B^{2} B^{*} \quad \alpha, \beta, \gamma \text { complexes, } c \text { réel }
$$

où les indices $t$ et $z$ sont relatifs aux variables de dérivation et le terme $\nabla_{l}^{2}$ est le Laplacien transverse, c'est à dire par rapport aux coordonnées $\mathrm{x}$ et $\mathrm{y}$.

La résolution numérique de cette équation avec des coefficients adaptés au laser prédit l'existence de défauts comme les structures spirales. Les structures effectivement observées dans les lasers en étant exemptes, il est apparu primordial de raffiner le modèle. L'élément-clé manquant dans celui-ci apparaît être la courbure des miroirs ${ }^{9}$. Celle-ci joue en effet un rôle essentiel dans la constitution du faisceau dans le laser. Des paramètres comme la taille du faisceau gaussien, l'espacement entre les modes transverses et leur géométrie en dépendent de façon cruciale. Les modèles correspondants sont actuellement étudiés analytiquement et numériquement et l'on devrait bientôt disposer de théories décrivant de façon satisfaisante les structures observées dans les lasers à grand nombre de Fresnel.

\section{ANNEXE 1: NATURE DES POINTS FIXES}

Les points fixes d'un système différentiel de type

$$
\frac{d x}{d t}=F(x)
$$

avec $\mathbf{x}=\left(\mathrm{x}_{1}, \mathrm{x}_{2}, \ldots . \mathrm{x}_{\mathrm{n}}\right)$, sont les points $\mathrm{x}_{\mathrm{F}}$ tels que

$$
F\left(x_{F}\right)=0
$$

Pour déterminer la stabilité de ces solutions stationnaires, on effectue une analyse de stabilité linéaire, c'est à dire que l'on pose

$$
x=x_{F}+\varepsilon
$$

et que l'on développe l'équation (A-1) en ne gardant que les termes d'ordre 1 en $\varepsilon$. On aboutit alors à une équation linéaire à $\mathrm{n}$ variables du type

$$
\frac{d}{d t}[\varepsilon]=(L)[\varepsilon]
$$

La solution de cette équation passe par la résolution de l'équation caractéristique d'ordre $\mathrm{n}$ de la matrice $(L)$

$$
\operatorname{det}(L-\lambda I)=0
$$

Suivant la valeur de n, le système (A-2) admet différents types de solutions.

9 Voir par exemple P. K. Jakobsen et al. Phys. Rev. A 49, 4189 (1994) et J. Lega et al. Phys. Rev. A 49, 4201 (1994). 
- cas monodimensionnel $(n=1)$

C'est le cas par exemple du laser de classe $\mathrm{A}$, dont la dynamique est régie par une seule équation (voir par exemple (7)). L'équation caractéristique est du premier degré et sa racine réelle. Le point fixe est instable si elle est positive, stable dans le cas contraire. Ces deux cas sont illustrés dans le diagramme ci-dessous correspondant à un laser au dessus du seuil.

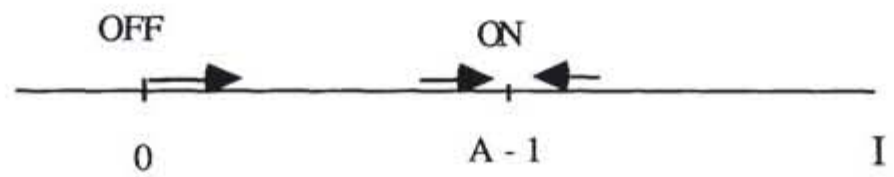

Figure A-1 : Dans un laser au dessus du seuil, le point $\mathrm{I}=0$ est répulseur et le point $\mathrm{I}=(\mathrm{A}-1)$ attracteur.

- cas bidimensionnel

La résolution de l'équation caractéristique mène dans ce cas à une équation du second degré, Selon la nature et le signe des solutions, différents comportements dynamiques sont obtenus

$$
\begin{array}{llll}
\text { - racines réelles } & \lambda_{1}, \lambda_{2}>0 & \text { point répulseur (instable) Fig. A-2 a } \\
& \lambda_{1}>0, \lambda_{2}<0 & \text { point col (instable) } & \text { Fig. A-2 b } \\
& \lambda_{1}<0, \lambda_{2}<0 & \text { point attracteur (stable) } & \text { Fig. A-2 c }
\end{array}
$$

- racines complexes $\lambda=\rho \pm i \omega$

$\begin{array}{lll}\rho>0 & \text { foyer instable } & \text { Fig. A-2d } \\ \rho<0 & \text { foyer stable } & \text { Fig. A-2 e }\end{array}$

Ces situations correspondent à des situations où le système évolue de façon oscillatoire autour de l'équilibre. Ces oscillations sont amorties si $\rho$ est négatif et divergentes dans le cas contraire.

\section{ANNEXE 2: ÉOUATIONS ADIMENSIONNÉES DU LASER}

Pour simplifier l'écriture, on discutera ici les équations du laser monomode en élargissement homogène en absence d'effets de diffraction. Ces équations sont classiquement mises sous la forme

$$
\begin{gathered}
\frac{d}{d t} E(t)=-\kappa E(t)-i \omega_{c} E(t)-i g P(t) \\
\frac{d}{d t} P(t)=-\gamma_{a b} P(t)-i \omega_{0} P(t)+i g^{*} E(t) N(t) \\
\frac{d}{d t} N(t)=-\gamma\left(N(t)-N_{0}\right)+2 i\left(g E^{*}(t) P(t)-g^{*} E(t) P^{*}(t)\right)
\end{gathered}
$$

où $\mathrm{N}(\mathrm{t})$ est la demi-différence de population entre niveaux supérieur et inférieur de la transition laser et $\mathrm{g}$ le paramètre d'interaction lumière-matière proportionnel à l'élément de matrice $\mathrm{d}$ du moment dipolaire électrique.

Par passage dans le référentiel tournant à la fréquence optique

$$
E(t)=E(t) e^{i\left(\omega t+\phi_{0}\right)}, P(t)=P(t) e^{i \omega x}, N(t)=-\left(N(t)-N_{0}\right),
$$

où le déphasage inclut à la fois le terme i et l'argument de l'élément de matrice complexe d'interaction 


$$
\phi_{0}=\frac{\pi}{2}-\arg (g)
$$

les équations intermédiaires s'écrivent de la façon suivante

$$
\begin{gathered}
\frac{d}{d t} E(t)=-\kappa E(t)-|g| P(t) \\
\frac{d}{d t} P(t)=-\gamma_{a b} P(t)+i\left(\omega-\omega_{0}\right) P(t)+\left|g / E(t) N_{O}(t)-\right| g \mid E(t) N(t) \\
\frac{d}{d t} N(t)=-\gamma N(t)+2 / g /\left(E^{*}(t) P(t)-E(t) P^{*}(t)\right)
\end{gathered}
$$

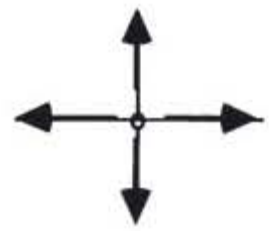

a

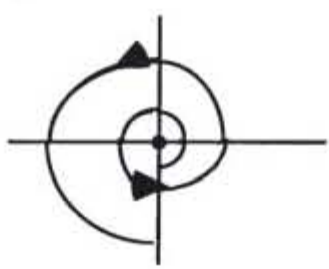

d

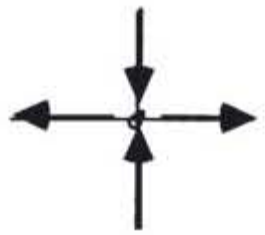

b

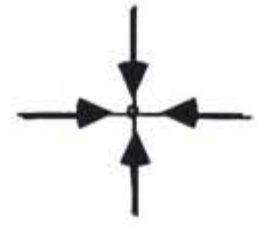

c suivant la nature des coefficients de stabilité. Coefficients réels a) pic, b) col c) puits ou attracteur ou complexes à partie réelle positive (d) ou négative (e).

A l'aide d'un changement de variables supplémentaire, on se ramène au système de Lorenz complexe

$$
\begin{gathered}
\frac{d}{d \tau} X(\tau)=-\sigma X(\tau)+\sigma Y(\tau) \\
\frac{d}{d \tau} Y(\tau)=-(1-i e) Y(\tau)+r X(\tau)-X(\tau) Z(\tau) \\
\frac{d}{d \tau} Z(\tau)=-b Z(\tau)+\frac{1}{2}\left(X^{*}(\tau) Y(\tau)-X(\tau) Y^{*}(\tau)\right) \\
e=\frac{\omega-\omega_{0}}{\gamma} \quad r=\frac{N_{0}}{N_{0}^{\text {seuil }}} \quad \text { où } \quad N \sigma^{\text {seuil }}=\frac{\kappa \gamma}{\mid g / 2}
\end{gathered}
$$


La seule différence avec le modèle de Lorenz complexe réside dans le fait que le paramètre de pompage normalisé $\mathrm{r}$ est ici purement réel.

\section{ANNEXE 3: CRITÈRE DE ROUTH-HURWITZ}

L'analyse de stabilité linéaire des solutions des systèmes de type (A-2) fait souvent un usage extensif du critère donné ci-dessous( critère de Routh-Hurwitz)

Théorème : Le polynôme $D_{k}(0)=s^{k}+a_{1} s^{k-1}+a_{2} s^{k-2}+\ldots .+a_{k-1} s+a_{k}$ est un polynôme de Hurwitz (c.a.d. que ses racines sont toutes à partie réelle négative) si et seulement si tous les mineurs de Hurwitz sont positifs soit

$$
\begin{aligned}
& D_{1}=a_{1} \quad D_{2}=\left|\begin{array}{cc}
a_{1} & a_{3} \\
1 & a_{2}
\end{array}\right| \\
& \text { - } \quad D_{3}=\left|\begin{array}{ccc}
a_{1} & a_{3} & a_{5} \\
1 & a_{2} & a_{4} \\
0 & a_{1} & a_{3}
\end{array}\right| \quad D_{4}=\left|\begin{array}{cccc}
a_{1} & a_{3} & a_{5} & a_{7} \\
1 & a_{2} & a_{4} & a_{6} \\
0 & a_{1} & a_{3} & a_{5} \\
0 & 1 & a_{2} & a_{4}
\end{array}\right| \quad \text { etc. }
\end{aligned}
$$

Examinons maintenant quelques cas simples, ceux des polynômes de degré faible.

a) Pour une équation du $2^{\mathrm{e}}$ degré $x^{2}-S x+P=0$

Ceci implique

$$
D_{1}=-S>0 \quad D_{2}=\left|\begin{array}{cc}
a_{1} & a_{3} \\
1 & a_{2}
\end{array}\right|=a_{1} a_{2}>0
$$

i.e. $-S . P>0$ soit $P>0$. On retrouve la condition classique pour que les racines d'un polynôme du second degré soient toutes deux négatives.

b) Pour une équation du $3^{\mathrm{e}}$ degré $\quad x^{3}+a_{1} x^{2}+a_{2} x+a_{3}=0$

$$
\begin{aligned}
& D_{1}=a_{1} \quad\left(a_{1}=-S\right) \quad>0 \\
& D_{2}=\left|\begin{array}{ll}
a_{1} & a_{3} \\
1 & a_{2}
\end{array}\right|=a_{1} a_{2}-a_{3}>0 \\
& D_{3}=\left|\begin{array}{lll}
a_{1} & a_{3} & a_{5} \\
1 & a_{2} & a_{4} \\
0 & a_{1} & a_{3}
\end{array}\right|=a_{1} a_{2} a_{3}-a_{3}^{2}=a_{3}\left(a_{1} a_{2}-a_{3}\right)>0
\end{aligned}
$$

i.e. en tenant compte de la condition sur $\mathrm{D}_{2}, a_{3}>0$. Remarquons qu'ici $a_{3}=-P$. Les conditions sur $\mathrm{D}_{1}$ et $\mathrm{D}_{3}$ sont donc triviales une fois trouvée la condition sur $\mathrm{D}_{2}$.

\section{ANNEXE 4: ANALOGIE MÉCANIOUE}

Le modèle monodimensionnel (ou laser de classe A) permet d'introduire une analogie avec le mouvement d'un point matériel dans un puits de potentiel. La loi fondamentale de la dynamique pour un mouvement dans un potentiel $\mathrm{V}(\mathrm{x})$ donne

$$
F=-\frac{d V}{d x}=+m \frac{d^{2} x}{d^{2}}
$$

Si l'on ajoute les forces de frottement visqueux $\left(-k \frac{d x}{d t}\right)$, on obtient

$$
m \frac{d^{2} x}{d t^{2}}+k \frac{d x}{d t}=-\frac{d V}{d x}
$$

Pour un mobile d'inertie négligeable $(\mathrm{m} \rightarrow 0)$, on aboutit à 


$$
\frac{\mathrm{dx}}{\mathrm{dt}}=-\frac{1}{\mathrm{k}} \frac{\mathrm{dV}}{\mathrm{dx}}
$$

Dans le cadre de l'approximation de faible saturation pour un laser de classe A (cf. éq.7),

$$
2 \frac{\mathrm{dE}}{\mathrm{dt}}=\alpha \mathrm{E}-\beta \mathrm{E}^{3}
$$

On peut extraire des deux dernières équations, le "potentiel" fonction du champ E du laser

$$
2 \frac{\mathrm{dV}}{\mathrm{dE}} \approx-\left[\alpha \mathrm{E}-\beta \mathrm{E}^{3}\right]
$$

soit

$$
2 \mathrm{~V}(\mathrm{E}) \approx-\left[\frac{\alpha \mathrm{E}^{2}}{2}-\frac{\beta \mathrm{E}^{4}}{4}\right]
$$

Les représentations graphiques de $\mathrm{V}(\mathrm{E})$ pour différentes valeurs de $\alpha$ sont données à la Figure A-3.
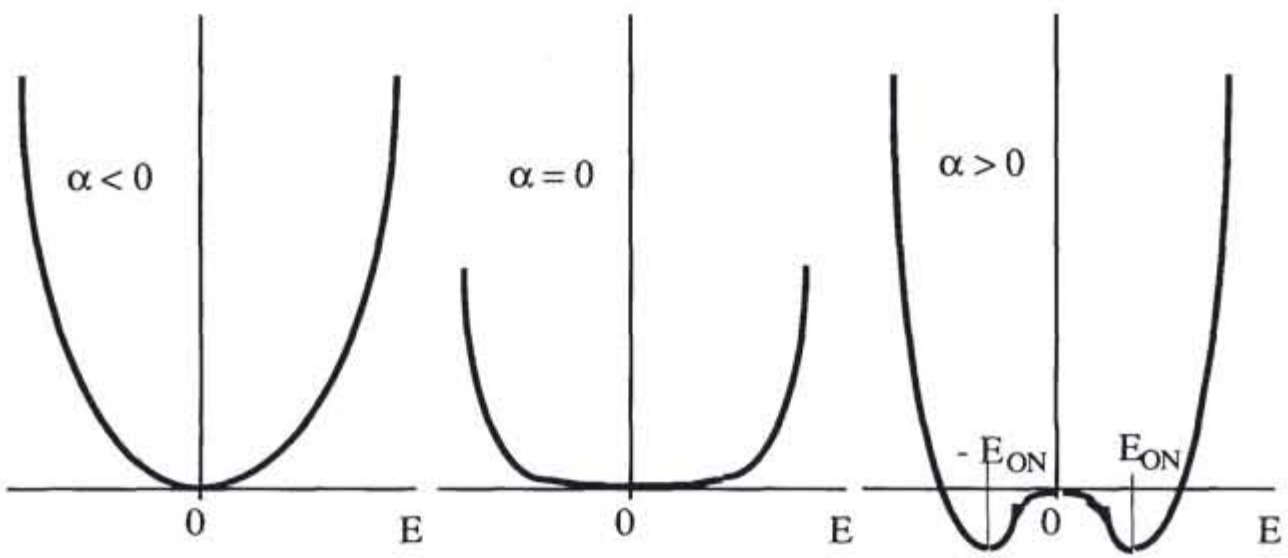

Figure A-3 : Formes du potentiel équivalent du laser sous le seuil $(\alpha<0)$, au seuil $(\alpha=0)$ et au dessus du seuil $(\alpha>0)$.

On peut retrouver certaines propriétés du laser grâce à l'analogie du mouvement d'un point matériel dans ce potentiel. Ainsi l'origine correspondant à la solution OFF est manifestement un point d'équilibre stable sous le seuil $(\alpha<0)$, instable au delà $(\alpha>0)$ tandis que la solution $\mathrm{ON}$ est stable dans tout son domaine d'existence. Le mouvement au voisinage de l'origine se ralentit de façon critique au seuil $(\alpha=0)$...

Il faut noter que cette analogie n'est valable que pour les lasers de classe A. Comme on l'a vu précédemment, dans les lasers de classe B, la dynamique fait intervenir l'inversion de population qui, ajoutant un degré de liberté, joue le rôle de l'inertie dans l'analogue mécanique. On voit ainsi qu'en présence d'inertie, le point peut effectuer des oscillations amorties avant d'atteindre l'équilibre. Moyennant certains ajustements, l'analogie reste encore valable.

Les effets de résonance amortie apparaissent de façon directe si l'on réécrit le système (3) sous forme d'une équation d'oscillateur en posant 
On obtient alors

$$
m=(A-1) e^{x}
$$

$$
\frac{d^{2} x}{d \tau^{2}}+\left[(A-I) e^{x}+1\right] \frac{d x}{d \tau}+\Omega_{r}^{2}\left(e^{x}-1\right)=0
$$

où $\Omega_{r}^{2}=G(A-1)$ est la pulsation qui est apparue dans l'analyse de stabilité linéaire. C'est l'équation d'un oscillateur nonlinéaire dans le potentiel de Toda

$$
V(x)=e^{x}-x
$$

On voit ainsi facilement que les oscillations de relaxation vers l'équilibre évoluent à une fréquence tendant vers zéro au seuil... 